\section{$\underset{\substack{\text { hommes } \\ \text { \& migrations }}}{ }$}

\section{Hommes \& migrations}

Revue française de référence sur les dynamiques

migratoires

\section{$1299 \mid 2012$}

Musulmanes et féministes en Grande-Bretagne

\title{
Le Musée de la mer à Gênes
}

Le nouveau pavillon "Mémoire et Migrations"

\section{Nicla Buonasorte et Anna Chiara Cimoli}

\section{Q OpenEdition \\ 1 Journals}

Édition électronique

URL : http://journals.openedition.org/hommesmigrations/1423

DOI : 10.4000/hommesmigrations. 1423

ISSN : 2262-3353

Éditeur

Musée national de l'histoire de l'immigration

Édition imprimée

Date de publication : 1 septembre 2012

Pagination : 123-127

ISSN : 1142-852X

\section{Référence électronique}

Nicla Buonasorte et Anna Chiara Cimoli, « Le Musée de la mer à Gênes », Hommes \& migrations [En

ligne], 1299 | 2012, mis en ligne le 29 mai 2013, consulté le 22 septembre 2020. URL : http:// journals.openedition.org/hommesmigrations/1423; DOI : https://doi.org/10.4000/

hommesmigrations. 1423

Ce document a été généré automatiquement le 22 septembre 2020.

Tous droits réservés 


\title{
Le Musée de la mer à Gênes
}

\author{
Le nouveau pavillon "Mémoire et Migrations"
}

\author{
Nicla Buonasorte et Anna Chiara Cimoli
}

1 L'Italie est un des pays européens les plus riches en musées consacrés aux migrations : on en compte presque une trentaine, et d'autres sont en projet. Ils sont consacrés à l'émigration en premier lieu, ce qui est compréhensible, si l'on considère qu'entre 1861 et 1985, 29 millions d'Italiens sont partis à l'étranger et que les départs - surtout du sud du pays vers la Suisse - ont continué jusqu'aux années soixante (ce n'est qu'en 1973 que l'Italie gagne un solde migratoire positif). L'émigration est donc une histoire encore "présente", presque superposée à celle de l'immigration. Cette pléthore de musées, sauf les exemples majeurs - Musée national de l'émigration italienne à Rome; Musée régional de l'émigration Pietro Conti à Gualdo Tadino, Musée Paolo Cresci pour l'histoire de l'émigration italienne à Lucca, Museo Narrante Nave della Sila à Camigliatello Silano -, est constituée par des petits avant-postes de mémoire, entre dimension privée et publique. D'autres expériences traitent le sujet des migrations d'une façon transversale et indirecte : c'est le cas, par exemple, des Musées du mineur de Casarano (Lecce) et de Fratta di Caneva (Pordenone) rendant un hommage aux travailleurs partis en Belgique, ou des nombreux écomusées qui racontent des histoires de voyages sans en faire leur focus principal. Ce n'est que depuis les années quatrevingt-dix que les musées italiens ont commencé à réfléchir à l'histoire de l'émigration et à se demander comment la documenter. Face à une forme de résistance dictée peutêtre par l'actualité politique récente, quelques musées commencent aujourd'hui à vouloir réfléchir à l'histoire de l'immigration de façon permanente. Les accents mis sur l'immigration, et donc sur l'identité, les deuxièmes générations, le multiculturalisme, etc., font l'objet depuis longtemps d'expériences "pionnières" menées par les départements éducatifs de quelques musées d'art contemporain (Castello di Rivoli et Fondazione Sandretto à Turin, MAMbo à Bologne, GAMeC à Bergame, entre autres), ou bien par des associations culturelles comme Askavusa, qui travaille au projet d'un musée en plein air sur l'île de Lampedusa où seraient exposés les objets trouvés dans le "cimetière des bateaux". Mais, au-delà de ces expériences ponctuelles, que font les 
musées ? Quelles questions se posent-ils par rapport à l'immigration, aux écoles, à la nouvelle composition démographique de la société?

\section{La migration au musée : une expérience grand public}

2 Le pavillon Mémoire et Migrations (MeM) est né d'une exposition temporaire de grand succès: La Merica! De Gênes à Ellis Island, le voyage par mer dans les années de l'émigration italienne. Ouverte au Galata-Musée de la mer de Gênes de 2008 à 2011, elle fut visitée par 400000 personnes. Le Galata est la construction la plus ancienne du port. Il fut restauré par l'architecte Guillermo Vazquez Consuegra. Il s'agit d'un bâtiment aux vastes surfaces en verre et aux terrasses plongeant sur le port, situé à côté de l'aquarium (l'une des attractions touristiques majeures en Italie). Les deux institutions ont été inaugurées en 2004, l'année où Gênes fut nommée capitale européenne de la culture. L'architecture du Galata évoque celle d'un navire et son grand mirador au dernier étage suggère déjà l'expérience du voyage transocéanique. Il est intéressant de remarquer que le MeM, institution muséale d'avant-garde pour le choix du sujet et la façon dont il le traite, fait partie d'un Musée de la mer avec une physionomie bien précise - c'est-à-dire avec un fort intérêt touristique et une approche "grand public" et non pas d'un musée de la ville, d'histoire, d'anthropologie. Est-ce que le lieu apparemment "neutre" et l'accent sur l'idée du voyage contrebalancent, du point de vue de la communication, les contenus explicites et les parti-pris parfois courageux de cette section? Bien sûr, pas de déclarations politiques, pas de provocations dans les salles : tout est soutenu par des données objectives, dont les sources sont toujours citées. Ainsi, dans le MeM, l'accent est mis sur la "normalité", sur l'idée d'une vie quotidienne partagée (à l'école, sur les lieux de travail, etc.). Le MeM est le premier pavillon muséal italien qui consacre une section importante à l'histoire de l'immigration. Ouvert en novembre 2011, il a depuis lors été visité par le président de la Chambre des députés Gianfranco Fini et par le ministre de la Coopération internationale et de l'Intégration Andrea Riccardi. L'affiche géante qui nous accueille dès l'entrée montre la photographie sépia d'un ancien navire sur lequel un jeune homme africain, chemise rouge et blue-jean, nous regarde dans les yeux. Un voyage par mer à la découverte du passé pour comprendre le présent, voilà le slogan sur l'affiche : le message est donc très clair et explicite dès le départ. L'objectif est de lier histoire et chronique, mémoire et défis contemporains, en essayant de jeter un pont sur les années d'oubli collectif que l'Italie a vécu depuis l'après-guerre jusqu'à très récemment, peutêtre trop occupée à vivre sa première, soudaine, saison de bien-être économique. Fruit de la réflexion conjointe du directeur du Musée de la mer, Pierangelo Campodonico, et de son équipe d'un côté, et de l'architecte Deborah Bruno de l'autre, le pavillon joue, dans la partie consacrée à l'émigration, la carte de l'empathie, de la "mise en situation", de la reconstruction d'ambiance. Le visiteur, qui reçoit avant l'entrée la copie d'un vrai passeport - selon le modèle des musées de la Shoah -, est appelé à s'identifier, à vivre une expérience, plutôt qu'à la "comprendre" de façon intellectuelle. Il ignore "son" destin avant le départ et il va le découvrir peu à peu à travers les photographies et les documents de voyage, jusqu'à connaître l'issue de son voyage, son succès ou bien son échec. Pas de panneaux de chiffres, pas de cartes du monde avec les routes des voyages, pas de longs textes à lire: les explications existent, bien sûr, mais l'invitation est à partir en voyage, physiquement et émotionnellement, main dans la main avec les émigrants. Ainsi, dans cette partie du MeM, les documents (lettres, textes, récits...) sont 
écrits et lus sous nos yeux par des acteurs habillés "à l'ancienne" qui apparaissent sur des moniteurs au lieu d'être montrés dans des vitrines. Dans la première salle, par exemple, on trouve la reconstruction d'un "caruggio" (les anciennes ruelles de Gênes), avec les maisons peintes de couleurs vives selon la tradition régionale. Penchés aux fenêtres-vidéos des maisons, des habitants-acteurs parlent entre eux et avec le voyageur, mélangeant lieux communs et recommandations.

\section{Le croisement des histoires}

3 Après le départ, le voyage : il est démontré que c'est à partir de l'exploitation des gains du transport des émigrants que les grandes compagnies maritimes ont pu développer les navires à vapeur et renouveler la flotte européenne. Les destinations classiques des Italiens au moment de la grande migration sont l'Argentine, le Brésil, les États-Unis. En Argentine, dans le quartier de La Boca de Buenos Aires, nous retrouvons une colonie italienne, ligure en particulier. La deuxième destination retrace une histoire presque oubliée, celle des Italiens au Brésil, entre plantations de café et forêt tropicale : une histoire qui a concerné presque deux millions de personnes, la plupart fermiers pauvres du nord de l'Italie, destinés à se substituer aux esclaves noirs récemment libérés. Beaucoup se retrouvèrent dans la forêt, au milieu d'Indios parfois hostiles, de bêtes sauvages, jusqu'au moment de l'exode rural. Ils se déplaceront ensuite dans les grandes villes comme São Paulo ou Porto Alegre, où ils vont s'insérer dans le melting pot de la société brésilienne. La troisième destination est "la Merica", par Ellis Island. Des grands vitraux du musée, on peut imaginer New York et entrevoir les paquebots italiens qui, pendant des décennies, transportèrent des millions d'émigrants : le Duilio et le Rex, parmi d'autres. Le visiteur doit obligatoirement passer le contrôle sanitaire et psychologique. On voit les visages d'autres émigrants, pour souligner qu'Ellis Island, "l'île des larmes", appartient à tout le monde, et non pas seulement aux Italiens. On aurait pu s'arrêter là, mais les migrations depuis et vers notre pays n'ont jamais cessé. L'histoire continue et, parfois, se répète. Le passage historique et physique de la partie consacrée à l'émigration à celle qui parle d'immigration est marqué par les photos d'Uliano Lucas, qui montrent les derniers émigrés italiens partis en Belgique et en Allemagne et les figures silencieuses et solitaires des premiers immigrés arrivés à Turin, Rome, Milan, Gênes - qui, même dans cette phase, joue le rôle de port de la migration - et aussi à Mazara del Vallo (Sicile), ou dans le riche Nord-Est. La section suivante est appelée "Cartes postales". On peut choisir une grande image qui, une fois posée sur la table, va déclencher une vidéo: du Sénégal, de l'Afghanistan, du Niger, quelqu'un est parti et nous raconte son voyage vers l'Occident. Mais les voyages ne finissent pas tous bien, au contraire... C'est ce que nous rappelle un des moments les plus intenses du parcours: le barcone ("bateau") de Lampedusa, une des premières embarcations à rejoindre la côte italienne chargée de réfugiés, telles les 55000 personnes débarquées en 2011. L'objet, le bateau, donne la dimension réelle de ces voyages, mais ne suffit pas à en communiquer la dimension tragique : pour cela furent ajoutés les images des sauvetages, les voix et les témoignages recueillis par les journalistes durant ces années. L'année dernière, l'écrivain Claudio Magris nous invitait à nous mettre dans la peau des immigrés. C'est ce que l'on a cherché à faire dans cette section: les voix, les bruits, les objets retrouvés dans les bateaux témoignent d'une réalité terrible à laquelle on s'est peu à peu habitués, parce que éloignée de nos yeux, parce que parfois faite de chiffres et non pas de personnes.Mais cela n'est qu'une 
facette de l'immigration en Italie, une tesselle dans la mosaïque. Lançant une provocation, les commissaires ont intitulé une section "Qui est-ce qui nous vole le travail?" Des programmes de la télévision italienne et des vidéos commandées pour l'occasion permettent de documenter, chiffres à l'appui, la dimension du travail. Aux immigrés sont souvent laissés les travaux les plus dangereux, payés en moyenne $20 \%$ de moins qu'aux Italiens; l'accent est mis ici sur le rôle des femmes immigrées dans le welfare state "à l'italienne" (soin des personnes âgées, des handicapés, à la maison, etc.).

\section{Apprendre autrement par l'empathie}

Autre dimension clé: l'école. Le visiteur, face à une "vraie" classe (une vidéo interactive), peut appeler un des étudiants qui lit un texte concernant sa vraie histoire. Il y parle de son sentiment d'égalité ou d'inégalité ressenti par rapport aux autres. Comme le montre l'histoire de la France, il s'agit de se préparer à répondre aux sollicitations des deuxièmes générations.

Si nous nous rapprochons un peu, nous rencontrons des personnes, des sentiments, des espoirs. Et voilà une grande vitrine pleine d'objets : ce sont les objets conservés par les migrants lorsqu'ils ont quitté leur pays, véritables ponts entre deux cultures et deux mondes différents, qui permettent de garder son identité même si l'on est à des milliers de kilomètres de chez soi. Le voyage aboutit en cuisine : chef Kumalé - alias Vittorio Castellani, journaliste et chef cuisinier -, avec l'aide de quatre chefs de différentes nationalités, nous montre comment préparer les plats les plus représentatifs de quelques traditions gastronomiques qui vont peut-être influencer nos propres goûts. À la fin du parcours, une série de niches rouges, les "confessionnaux de la réflexion", abritent des écrans où les conservateurs du musée posent dix questions sur les lieux communs les plus répandus. Entre quizz et sondage, cette sorte d'auto-examen, avec un clin d'œil ironique aux reality shows, vise à vérifier ce que nous connaissons réellement des migrations. C'est le moment d'un bilan avant de sortir, parce que le monde a changé, mais peut-être que nous, nous n'en sommes pas conscients. Ce parcours impose quelques réflexions sur les choix muséologiques. Le point de départ des conservateurs a été le constat de l'insuffisance des photographies, des documents ou des fresques historiques. Pour parler de l'émigrant, il faudrait le faire parler avec ses propres mots. Cela a demandé un long et patient travail de collecte de lettres, de récits autobiographiques, de témoignages sur l'émigration italienne et de transformation en témoignages oraux, en récits racontés à l'oreille du spectateur. Dans ce but, on a fait appel aux techniques théâtrales et à des acteurs professionnels. Ainsi le document ne reste pas muet, mais parle, s'exprime. On a utilisé des registres communicatifs différents, toujours en pensant que le défi d'emmener un Européen du XXI ${ }^{\mathrm{e}}$ siècle dans la queue pour l'embarquement, de lui faire vivre l'humiliation d'un dortoir de troisième classe à l'intérieur d'un paquebot, ou de lui faire subir l'interrogatoire d'un fonctionnaire américain de l'Immigration Service, était une occasion d'expérience personnelle, et même un risque, à ne pas rater. La recherche sur l'émigration du XIX ${ }^{e}$ siècle à nos jours est pour l'historien une action sur la chair et sur la mémoire. À partir de la réflexion et d'une meilleure compréhension du phénomène migratoire dans tous ses aspects naît une conscience personnelle à l'égard d'un des phénomènes les plus importants de nos temps. Aux muséologues est dévolue la tâche délicate et importante 
de réaliser une sorte de "courroie de transmission" entre ceux qui produisent le savoir historique (les historiens, les chercheurs, les instituts spécialisés, etc.) et le grand public. Pour cela fut choisi le modèle de la reconstitution d'ambiances, c'est-à-dire la reconstruction de milieux immersifs qui peuvent aider à lire une histoire, à l'imaginer, à la vivre. Le MeM a pour but d'être compris par tous, et pour cela il joue la carte des émotions : il veut communiquer à travers l'empathie afin que le visiteur ne se sente pas comme un observateur de l'histoire, mais un protagoniste qui la vit dans sa chair.

\section{AUTEURS}

NICLA BUONASORTE

Curatrice du MeM.

ANNA CHIARA CIMOLI

MeLa project-Politecnico di Milano. 